\title{
Aquatic Plant Diversity of Ponds in Thrissur District, Kerala, India
}

\author{
P. Tessy Paul \\ Department of Botany, Christ College (Autonomous), Irinjalakuda, Thrissur-680 125, India \\ E-mail:dr.tessypaul@gmail.com
}

\begin{abstract}
The analysis of aquatic plant diversity was conducted in twenty ponds of Thrissur district, Kerala, during April 2014 to March 2016. During the period of study, 41 macrophytes were identified which comes under 35 genera belonging to 24 families including Pteridophytes. $46.3 \%$ of total plant species that were recorded fall into the category of marshy, followed by $19.5 \%$ submerged hydrophytes, $12.2 \%$ emergent hydrophytes, $12.2 \%$ free floating hydrophytes and $9.8 \%$ attached floating hydrophytes. The diverse plant family is Poaceae followed by Hydrocharitaceae, Onagraceae and Asteraceae.
\end{abstract}

Keywords: Aquatic plants, Macrophytes, Thrissur, Pteridophytes, Poaceae, Hydrocharitaceae, Onagraceae, Asteraceae

The macrophytes play a vital role in healthy aquatic ecosystems and serve as primary producers of oxygen through photosynthesis. These plants supply a wide variety of nesting habitats for aquatic organisms. They provide a substrate for epiphytic algae and shelter for many invertebrates, aid in nutrient cycling to and from the sediments and help to stabilize river and stream banks. The macroscopic flora includes the aquatic Angiosperms (flowering plants) and Pteridophytes (ferns). The macrophytes constitutes a diverse assemblage of taxonomic groups and are classified into four categories based on their habit of growth namely free floating, attached floating, submerged and emergent (Kumar 2015). Majority of the ponds in India are shallow and perennial freshwater bodies. Algae in deep waters dominate the aquatic biota and macrophytes dominate in the shallow waters. The macrophytes are the major contributors of pond productivity. The aquatic macro-vegetation plays an important role in maintaining ecological balance (Bhagyaleena and Gopalan 2012). The eutrophic water bodies are characterized by the presence of aquatic plants (Bronmark and Hansson 2017). The increased number of macro vegetation indicates the eutrophic condition of the reservoirs (Reju et al 2015). The macrophytes produce additional amount of nutrients and that pollute the water after their death (Tewari and Mittal 2020). The water quality of the freshwater bodies is rapidly declining (Nandal et al 2020). The aquatic plants in the freshwater habitats of India were reported by Cook (1996). The baseline data on biodiversity is essential for the conservation and management strategies of wetland and aquatic habitats (Ravi et al 2020). In Kerala the systematic reports regarding the freshwater ecology, limnology and conservation biology of ponds was insufficient.

\section{MATERIAL AND METHODS}

The present work was conducted in twenty ponds $\left(P_{1}-\right.$ $\mathrm{P}_{20}$ ) of Thrissur district, Kerala, during April 2014 to March 2016 (Table 1). The twigs of plants were collected periodically with flowers and fruits. The habit, morphology and floristic characters are recorded during the time of collection. The plants collected were identified with the help of floras (Manilal and Sivarajan 1982, Gamble and Fischer 1915 - 1936, Sreekumar and Nair 1991).

\section{RESULTS AND DISCUSSION}

During the period of study, 41 macrophytes were identified which comes under 35 genera belonging to 24 families including Pteridophytes (Table 2 and Plate 1). The $46.3 \%$ of total plant species that were recorded fall into the category of marshy land, followed by $19.5 \%$ submerged hydrophytes, $12.2 \%$ emergent hydrophytes, $12.2 \%$ free floating hydrophytes and $9.8 \%$ attached floating hydrophytes (Fig. 1). The diverse family is Poaceae followed by Hydrocharitaceae, Onagraceae and Asteraceae (Table 2). The free floating hydrophytes found in the ponds of Thrissur district are Eichhornia crassipes (Mart.) Solms, Lemna perpusilla Torrey, Pistia stratiotes L., Azolla pinnata R. Br. and Salvinia molesta D. Mitch. The rooted plants with floating leaves are Nymphaea nouchali Burm. f., Nymphaea rubra Roxb. ex Salisb., Nymphoides hydrophylla (Lour.) O. Ktze. and Nymphoides indica (L.) O. Ktze. The prominent growth of the floating plants will not allow light to pass through the water and the growth of the microphytes are inhibited. The rooted submerged plants found in the ponds are Ottelia alismoides (L.) Pers., Utricularia aurea Lour., Cabomba caroliniana Gray., Hydrilla verticillata (L. f.) Royle, Ceratophyllum 
Table 1. GPS locations of the ponds studied

\begin{tabular}{|c|c|c|}
\hline $\begin{array}{l}\text { Pond } \\
\text { No. }\end{array}$ & Name of the pond & GPS location \\
\hline p & Pond at Christ College campus, Irinjalakuda & $\begin{array}{l}10^{\circ} 21^{\prime} 23.4^{\prime \prime} \mathrm{N} \\
76^{\circ} 12^{\prime} 44.1^{\prime \prime} \mathrm{E}\end{array}$ \\
\hline $\mathrm{P}_{2}$ & Njourikulam, Irinjalakuda & $\begin{array}{l}10^{\circ} 20^{\prime} 53.1 " \mathrm{~N} \\
76^{\circ} 12^{\prime} 48.3^{\prime \prime} \mathrm{E}\end{array}$ \\
\hline $\mathrm{P}_{3}$ & Andanikulam, Irinjalakuda & $\begin{array}{l}10^{\circ} 18^{\prime} 56.2^{\prime \prime} \mathrm{N} \\
76^{\circ} 13^{\prime} 12.5^{\prime \prime} \mathrm{E}\end{array}$ \\
\hline $\mathrm{P}_{4}$ & Marathampully pond, Irinjalakuda & $\begin{array}{l}10^{\circ} 20^{\prime} 29.8^{\prime \prime} \mathrm{N} \\
76^{\circ} 12^{\prime} 55.0^{\prime \prime} \mathrm{E}\end{array}$ \\
\hline$P_{5}$ & Pallikulam, Thrissur & $\begin{array}{l}10^{\circ} 31 ' 17.5 " \mathrm{~N} \\
76^{\circ} 13^{\prime} 20.4 " \mathrm{E}\end{array}$ \\
\hline $\mathrm{P}_{6}$ & Ayyanpadi pond, Edamuttam & $\begin{array}{l}10^{\circ} 22^{\prime} 32.7^{\prime \prime} \mathrm{N} \\
76^{\circ} 08^{\prime} 10.3^{\prime \prime} \mathrm{E}\end{array}$ \\
\hline $\mathrm{P}_{7}$ & Pond at Edamuttam & $\begin{array}{l}10^{\circ} 222^{\prime} 29.1 " \mathrm{~N} \\
76^{\circ} 07 \cdot 33.1^{\prime \prime} \mathrm{E}\end{array}$ \\
\hline$P_{8}$ & Kothakulam pond, Valapad & $\begin{array}{l}10^{\circ} 23^{\prime} 40.7^{\prime \prime N} \\
76^{\circ} 06^{\prime} 58.5^{\prime \prime} \mathrm{E}\end{array}$ \\
\hline $\mathrm{P}_{9}$ & Kuttankulam, Irinjalakuda & $\begin{array}{l}10^{\circ} 20^{\prime} 48.3^{\prime \prime} \mathrm{N} \\
76^{\circ} 12^{\prime} 10.0^{\prime \prime} \mathrm{E}\end{array}$ \\
\hline$P_{10}$ & Temple pond, Kodungallur & $\begin{array}{l}10^{\circ} 13^{\prime} 38.0^{\prime \prime} \mathrm{N} \\
76^{\circ} 11^{\prime} 52.2^{\prime \prime} \mathrm{E}\end{array}$ \\
\hline$P_{11}$ & Temple pond, Guruvayur & $\begin{array}{l}10^{\circ} 35^{\prime} 44.3^{\prime \prime} \mathrm{N} \\
76^{\circ} 02^{\prime} 21.3^{\prime \prime} \mathrm{E}\end{array}$ \\
\hline$P_{12}$ & Vadakkechira temple pond, Thrissur & $\begin{array}{l}10^{\circ} 31^{\prime} 46.1 " \mathrm{~N} \\
76^{\circ} 12^{\prime} 57.9^{\prime \prime} \mathrm{E}\end{array}$ \\
\hline $\mathrm{P}_{13}$ & $\begin{array}{l}\text { Pond at Kaduppassery, Velukkara } \\
\text { Panchayath }\end{array}$ & $\begin{array}{l}10^{\circ} 18^{\prime} 59.8^{\prime \prime} \mathrm{N} \\
76^{\circ} 15^{\prime} 15.5^{\prime \prime} \mathrm{E}\end{array}$ \\
\hline $\mathrm{P}_{14}$ & Oomenkulam, Irinjalakuda & $\begin{array}{l}10^{\circ} 20^{\prime} 26.5^{\prime \prime} \mathrm{N} \\
76^{\circ} 13^{\prime} 06.9^{\prime \prime} \mathrm{E}\end{array}$ \\
\hline$P_{15}$ & Velathikulam, Muriyad Panchayath & $\begin{array}{l}10^{\circ} 21^{\prime} 07.4^{\prime \prime} \mathrm{N} \\
76^{\circ} 13^{\prime} 49.2^{\prime \prime} \mathrm{E}\end{array}$ \\
\hline$P_{16}$ & Ayyappankavu kulam, Cheloor, Irinjalakuda & $\begin{array}{l}10^{\circ} 20^{\prime} 22.6^{\prime \prime} \mathrm{N} \\
76^{\circ} 11^{\prime} 40.2^{\prime \prime} \mathrm{E}\end{array}$ \\
\hline$P_{17}$ & Kottamkulam, Thalikulam & $\begin{array}{l}10^{\circ} 26^{\prime} 07.7^{\prime \prime} \mathrm{N} \\
76^{\circ} 05^{\prime} 49.11^{\prime \prime} \mathrm{E}\end{array}$ \\
\hline$P_{18}$ & Narayanamangalam temple pond, Palakkal & $\begin{array}{l}10^{\circ} 28^{\prime} 12.1^{\prime \prime} \mathrm{N} \\
76^{\circ} 13^{\prime} 14.4^{\prime \prime} \mathrm{E}\end{array}$ \\
\hline $\mathrm{P}_{19}$ & Pallikulam, Koratty & $\begin{array}{l}10^{\circ} 16^{\prime} 10.3^{\prime \prime N} \\
76^{\circ} 20^{\prime} 39.9^{\prime \prime} \mathrm{E}\end{array}$ \\
\hline$P_{20}$ & Mudichira, Muriyad Panchayath & $\begin{array}{l}10^{\circ} 21^{\prime} 12.3^{\prime \prime} \mathrm{N} \\
76^{\circ} 14^{\prime} 00.6^{\prime \prime} \mathrm{E}\end{array}$ \\
\hline
\end{tabular}

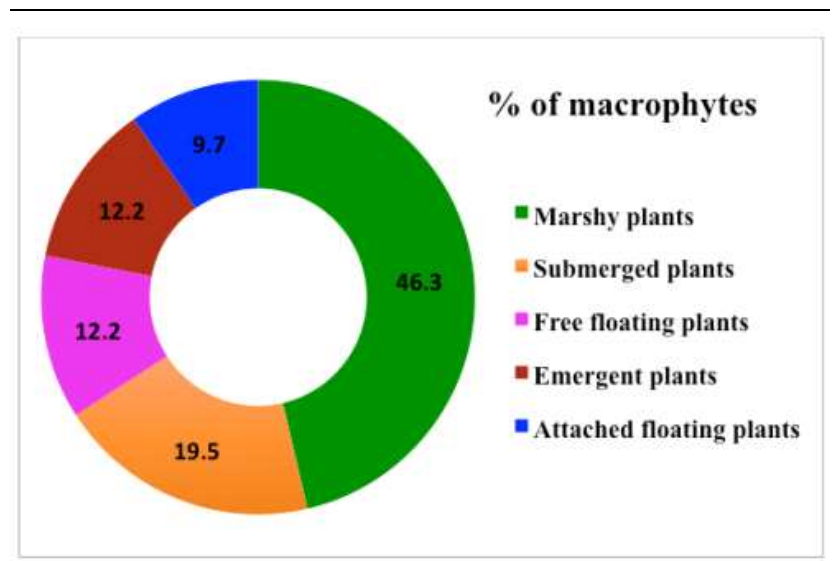

Fig. 1. Distribution of macrophytes in the ponds of Thrissur district

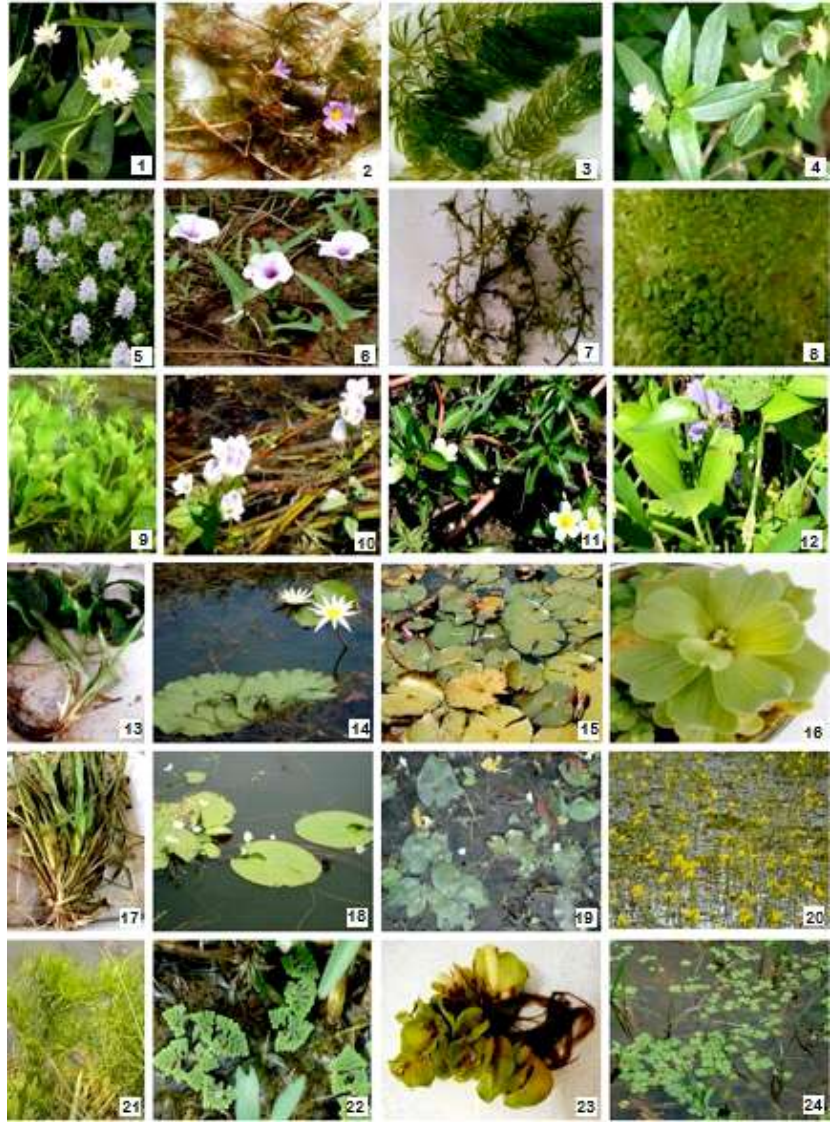

Plate 1. 1. Alternanthera philoxeroides (Mart.) Grisb.; 2. Cabomba caroliniana Gray.; 3. Ceratophyllum demersum L.; 4. Eclipta prostrata L.; 5. Eichhornia crassipes (Mart.) Solms.; 6. Ipomoea aquatica Forssk.; 7. Hydrilla verticillata (L. f.) Royle; 8. Lemna perpusilla Torrey; 9. Limnocharis flava (L.) Buch.; 10. Limnophila aquatica (Roxb.) Alston; 11. Ludwigia adscendens (L.) Hara.; 12. Monochoria vaginalis (Burm. f.) Presl; 13. Ottelia alismoides (L.) Pers.; 14. Nymphaea nouchali Burm. f.; 15. Nymphaea rubra Roxb. ex Salisb.; 16. Pistia stratiotes L.; 17. Vallisneria natans (Lour.) Hara; 18. Nymphoides indica (L.) O. Ktze.; 19. Nymphoides hydrophylla (Lour.) O. Ktze.; 20. Utricularia aurea Lour.; 21. Ceratopteris thalictroides (L.) Brongn.; 22. Azolla pinnata R. Br.; 23. Salvinia molesta D. Mitch.; 24. Marsilea minuta L.

demersum L., Najas graminea Del. and Najas indica (Willd.) Cham. The Cyperus pilosus Vahl., Cyperus compressus L. and Eragrostis unioloides (Retz.) Nees ex Steud were found to be dominant during dry season of the year in the marshy areas of the ponds.

The macrophytes have capacity to improve the water quality by absorbing nutrients, with their effective root system. At the same time death and decay of the macrophytes increases nutrient concentration and it leads to the eutrophication. Eichhornia crassipes (Mart.) Solms and 
Table 2. Distribution of macrophytes in the ponds of Thrissur district, Kerala

\begin{tabular}{|c|c|c|c|}
\hline Name of species & Family name & Habit & Pond No: \\
\hline Alternanthera philoxeroides (Mart.) Grisb. & Amaranthaceae & M & $\mathrm{P}_{13,}, \mathrm{P}_{14}$ \\
\hline Cabomba caroliniana Gray. & Cabombaceae & $\mathrm{S}$ & $\mathrm{P}_{13}$ \\
\hline Ceratophyllum demersum L. & Ceratophyllaceae & S & $\mathrm{P}_{1}$ \\
\hline Colocasia esculenta (L.) Schott & Araceae & $\mathrm{E}$ & $\mathrm{P}_{13,}, \mathrm{P}_{20}$ \\
\hline Cyperus compressus L. & Cyperaceae & M & $P_{1}, P_{2}, P_{3,} P_{13}, P_{15}, P_{16}, P_{17}, P_{20}$ \\
\hline Cyperus pilosus Vahl. & Cyperaceae & M & $P_{1}, P_{15}, P_{17}, P_{20}$ \\
\hline Echinochloa colona (L.) Link. & Poaceae & M & $\mathrm{P}_{2}$ \\
\hline Eclipta prostrata (L.) L. & Asteraceae & M & $P_{1}, P_{13}, P_{20}$ \\
\hline Eichhornia crassipes (Mart.) Solms. & Pontederiaceae & FF & $P_{6}, P_{13}$ \\
\hline Eragrostis unioloides (Retz.) Nees ex Steud & Poaceae & M & $P_{4}, P_{13}, P_{15}, P_{20}$ \\
\hline Hydrilla verticillata (L. f.) Royle & Hydrocharitaceae & $S$ & $P_{1}, P_{2}, P_{6,}, P_{13}, P_{14}, P_{18}$ \\
\hline Hygrophila schulli (Buch - Ham) M.R. \& S.M. Almeida & Acanthaceae & $E$ & $P_{2,} P_{6}, P_{13}, P_{20}$ \\
\hline Hygroryza aristata (Retz.) Nees ex Wight \& Arn. & Poaceae & $E$ & $P_{13}, P_{15}$ \\
\hline Ipomoea aquatica Forssk. & Convolvulaceae & M & $\mathrm{P}_{6}, \mathrm{P}_{13}$ \\
\hline Lemna perpusilla Torrey & Lemnaceae & FF & $\mathrm{P}_{7}, \mathrm{P}_{15}$ \\
\hline Limnocharis flava (L.) Buch. & Alismataceae & $E$ & $\mathrm{P}_{6}, \mathrm{P}_{13}$ \\
\hline Limnophila aquatica (Roxb.) Alston & Scrophulariaceae & $\mathrm{E}$ & $P_{13}$ \\
\hline Ludwigia adscendens (L.) Hara. & Onagraceae & M & $\mathrm{P}_{2}$ \\
\hline Ludwigia hyssopifolia (G. Don) Exell & Onagraceae & M & $\mathrm{P}_{13,} \mathrm{P}_{15}$ \\
\hline Ludwigia perennis L. & Onagraceae & M & $P_{2}, P_{4,}, P_{6,}, P_{12}, P_{13}, P_{15}$ \\
\hline Monochoria vaginalis (Burm. F.) Presl & Pontederiaceae & M & $P_{1}, P_{2}, P_{4}$ \\
\hline Najas graminea Del. & Najadaceae & S & $\mathrm{P}_{1}$ \\
\hline Najas indica (Willd.) Cham. & Najadaceae & S & $P_{1}$ \\
\hline Nymphaea nouchali Burm. F. & Nympaeaceae & $\mathrm{AF}$ & $P_{2,}, P_{8}, P_{10}, P_{11}, P_{14}$ \\
\hline Nymphaea rubra Roxb. Ex Salisb. & Nympaeaceae & $\mathrm{AF}$ & $P_{5,} P_{6}$ \\
\hline Nymphoides hydrophylla (Lour.) O. Ktze. & Menyanthaceae & $\mathrm{AF}$ & $\mathrm{P}_{2}$ \\
\hline Nymphoides indica (L.) O. Ktze. & Menyanthaceae & $\mathrm{AF}$ & $\mathrm{P}_{2}$ \\
\hline Ottelia alismoides (L.) Pers. & Hydrocharitaceae & S & $\mathrm{P}_{6}$ \\
\hline Panicum repens $\mathrm{L}$. & Poaceae & M & $P_{2}, P_{3}, P_{9}, P_{13}, P_{14}, P_{15}, P_{16}, P_{20}$ \\
\hline Paspalum scrobiculatum L. & Poaceae & M & $\mathrm{P}_{13,}, \mathrm{P}_{20}$ \\
\hline Pistia stratiotes L. & Araceae & FF & $P_{17}$ \\
\hline Persicaria barbata (L.) Hara var. barbata Joseph & Polygonaceae & M & $\mathrm{P}_{13}$ \\
\hline Sacciolepis interrupta (Willd.) Stapf. & Poaceae & M & $P_{9}$ \\
\hline Spilanthes ciliata HBK & Asteraceae & M & $\mathrm{P}_{13}$ \\
\hline Utricularia aurea Lour. & Lentibulariaceae & S & $\mathrm{P}_{1}, \mathrm{P}_{13}$ \\
\hline Vallisneria natans (Lour.) Hara & Hydrocharitaceae & S & $\mathrm{P}_{13}$ \\
\hline Wedelia chinensis (Osbeck) Merr & Asteraceae & M & $\mathrm{P}_{13}$ \\
\hline Azolla pinnata $\mathrm{R}$. Br. & Azollaceae & $\mathrm{FF}$ & $\mathrm{P}_{13}$ \\
\hline Ceratopteris thalictroides (L.) Brongn. & Parkeriaceae & M & $P_{4,}, P_{6,}, P_{13,}, P_{14,} P_{15,}, P_{17,}, P_{20}$ \\
\hline Marsilea minuta L. & Marsileaceae & M & $P_{4,} P_{6,} P_{13,} P_{20}$ \\
\hline Salvinia molesta D. Mitch. & Salviniaceae & FF & $P_{1}, P_{2}, P_{4,}, P_{12}, P_{13}, P_{19}$ \\
\hline
\end{tabular}

$\mathrm{AF}$ - Attached floating; E - Emergent; FF - Free floating; M - Marshy; S - Submerged 
Hydrilla verticillata (L.f.) Royle are capable in improving water quality by reducing nutrient concentration. The macrophytes such as Hydrilla, Ceratophyllum and Eichhornia grown in ponds have the ability to maintain water quality (Pradeep and Dwivedi 2016). Some Pteridophytes such as Azolla pinnata R. Br., Ceratopteris thalictroides (L.) Brongn., Marsilea minuta L. and Salvinia molesta D. Mitch. were also found abundant in the perrennial ponds studied (Table 2). Aquatic macrophytes act as microhabitat for the fauna of wetlands due to the diversity of growth forms which outcomes in a greater niche modification (Malaiya 2015). It is very important to conserve the natural ecosystems that are deteriorating at an alarming rate otherwise the remnants will significantly lose their ability to sustain the present biological diversity (Gupta et al 2020).

\section{CONCLUSION}

This assessment on the macrophytes in the perennial ponds of Thrissur district, Kerala is a comprehensive floristic analysis of the aquatic flora. During the investigation 41 macrophytes were reported which comes under 35 genera belonging to 24 families including Pteridophytes. This report will be useful for analyzing the biodiversity of the macrophytes in the ponds of Kerala. The ponds promote abundance and high richness of aquatic plants and enhance regional biodiversity. The aquatic macro-vegetation plays a significant role in maintaining ecological balance by nutrient recycling. The ponds that are not periodically cleaned showed more diversity of macrophytes.

\section{ACKNOWLEDGEMENT}

University Grants Commission, New Delhi is gratefully acknowledged for the Minor Research Project awarded to the author.

\section{REFERENCES}

Bhagyaleena P and Gopalan R 2012. Aquatic plant diversity of ponds in Nenmara panchayath, Palakkad district, Kerala, India. International Journal of Scientific and Research Publications 2(8): 1-3.

Bronmark C and Hansson LA 2017. The biology of lakes and ponds. Oxford University Press, UK, p 368.

Cook CDK 1996. Aquatic and wetland plants of India. Oxford University Press Inc., New York, p 394.

Gamble JS and Fischer CEC 1915-1936. The flora of Presidency of Madras, Vol. 1-3, Botanical Survey of India, Calcutta, $p$ 1389.

Gupta P, Tamot S and Shrivastava VK. 2020. Effect of physicochemical properties of water on macrophyte diversity with special reference to Bhoj Wetland. Indian Journal of Ecology 47(3): 764-768.

Kumar A 2015. Freshwater plankton and macrophytes of India. Daya Publishing House, Delhi, p 362.

Malaiya PS 2015. Study of aquatic weeds in Marpha pond of Anuppur, Madhya Pradesh. International Journal for Research in Applied Science and Engineering Technology 3(12): 281284.

Manilal KS and Sivarajan VV 1982. Flora of Calicut. Bishan Singh and Mahendra pal Singh, Co. Dehradun, p 387.

Nandal A, Kaushik N, Yadav SS, Rao AS, Singh N and Gulia SS 2020. Water quality assessment of pond water of Kalanaur Block, Rohtak, Haryana. Indian Journal of Ecology 47(1): 1-6.

Pradeep S and Dwivedi HS 2016. Diversity of aquatic macrophytes of Govardhan Sagar water body at Ujjain (M.P.) India. International Journal of Advanced Research in Biological Sciences 3(8): 89-93.

Ravi V, Samimalaimurugan K, Kalpana $\mathrm{P}$, Vijayakanth $\mathrm{P}$ and Ramamoorthy R 2020. Wetland and aquatic Angiosperm flora of Denkanikottai, Krishnagiri, Tamil Nadu. Indian Journal of Ecology 47(4): 1038-1043.

Reju J, Thampiraj S and Lawrence B 2015. Aquatic and marshy plants of four selected ponds in Venganoor Grama Panchayat, Trivandrum District, Kerala, India. International Research Journal of Biological Sciences 4(4): 49-51.

Sreekumar PV and Nair VJ 1991. Flora of Kerala: Grasses, Flora of India-Series 2. Botanical Survey of India, Calcutta, p 470.

Tewari V and Mittal DK 2020. Ecological impact of macrophytes on some freshwater bodies in Dehradun District, Indian Journal of Applied Research 10(7): 12-13. 